\title{
On the complexity of computing the handicap of a sufficient matrix
}

\author{
Etienne de Klerk • Marianna E. -Nagy
}

Received: 15 February 2010 / Accepted: 20 February 2011 / Published online: 4 June 2011

(C) The Author(s) 2011. This article is published with open access at Springerlink.com

\begin{abstract}
The class of sufficient matrices is important in the study of the linear complementarity problem (LCP)—some interior point methods (IPM's) for LCP's with sufficient data matrices have complexity polynomial in the bit size of the matrix and its handicap.In this paper we show that the handicap of a sufficient matrix may be exponential in its bit size, implying that the known complexity bounds of interior point methods are not polynomial in the input size of the LCP problem. We also introduce a semidefinite programming based heuristic, that provides a finite upper bond on the handicap, for the sub-class of $\mathcal{P}$-matrices (where all principal minors are positive).
\end{abstract}

Keywords Handicap of a sufficient matrix - Linear complementarity problem (LCP) $\cdot$ Semidefinite programming

Mathematics Subject Classification (2010) 90C33 Complementarity and equilibrium problems; variational inequalities (finite dimensions) .

90C22 Semidefinite Programming

\section{Introduction}

The linear complementarity problem (LCP) is defined as: Given $M \in \mathbb{R}^{n \times n}$ and $q \in \mathbb{R}^{n}$, find $x \in \mathbb{R}^{n}$ and $s \in \mathbb{R}^{n}$ such that

$$
-M x+q=s, \quad x_{i} \geq 0, \quad s_{i} \geq 0, \quad x_{i} s_{i}=0 \quad(i=1, \ldots, n) .
$$

E. de Klerk $(\varangle) \cdot$ M. E.-Nagy

Department of Econometrics and OR, Tilburg University,

Warandelaan 2, 5000 LE Tilburg, The Netherlands

e-mail: e.deklerk@uvt.nl

M. E.-Nagy

e-mail: m.nagy@uvt.nl 
Kojima et al. [7] showed that interior point methods may be used to solve the linear complementarity problem if $M$ belongs to a certain class of matrices, called $\mathcal{P}_{*}(\kappa)$ matrices.

Definition 1 Let $\kappa \geq 0$ be a nonnegative number. A matrix $M \in \mathbb{R}^{n \times n}$ is a $\mathcal{P}_{*}(\kappa)$ matrix if for all $x \in \mathbb{R}^{n}$

$$
(1+4 \kappa) \sum_{i \in \mathcal{I}_{+}(x)} x_{i}(M x)_{i}+\sum_{i \in \mathcal{I}_{-}(x)} x_{i}(M x)_{i} \geq 0,
$$

where $\mathcal{I}_{+}(x)=\left\{1 \leq i \leq n: x_{i}(M x)_{i}>0\right\}$ and $\mathcal{I}_{-}(x)=\left\{1 \leq i \leq n: x_{i}(M x)_{i}<\right.$ $0\}$.

The infimum value of $\kappa \geq 0$ such that $M$ is $\mathcal{P}_{*}(\kappa)$ is called the handicap of $M$, and is denoted by $\hat{\kappa}(M)$, i.e.

$$
\hat{\kappa}(M):=\inf \left\{\kappa \mid M \in \mathcal{P}_{*}(\kappa)\right\} .
$$

Matrices with finite handicap are known as $\mathcal{P}_{*}$-matrices or as sufficient matrices; see Sect. 2.1 for more details.

The worst-case iteration complexity of interior point methods depends on the parameter $\kappa \geq \hat{\kappa}(M)$. For example, the iteration complexity of the predictorcorrector algorithm by Potra and Liu [11] is $O((1+\kappa) \sqrt{n} L(M, q))$ in the Turing model of computation, where $L(M, q)$ is the bit size of the (integer) problem data $M \in \mathbb{Z}^{n \times n}$ and $q \in \mathbb{Z}^{n}$; see also Corollaries 6.7 and 6.9 in [7]. In this paper we show that the handicap may be exponential in the bit size of $M$. This implies that the iteration bound is not polynomial in $L(M, q)$. In particular, it implies that there are still no known polynomial-time algorithms (in the Turing model) to solve the class of LCP problems where $M$ is a $\mathcal{P}_{*}(\kappa)$-matrix.

It is of practical interest to decide if a given matrix $M$ is $\mathcal{P}_{*}(\kappa)$, for some given value $\kappa$. Indeed:

- some interior point algorithms require the value of $\kappa$, e.g. the method of Potra and Liu [11];

- some rounding schemes for LCP that convert $\epsilon$-optimal solutions to exact solutions require the value of $\kappa$, e.g. the rounding scheme in [5]. Here, the required value of $\epsilon$ depends on $\kappa$.

Of course, from a purely linear algebra viewpoint, it is of independent interest to compute or bound the handicap of a given matrix; see e.g. [16].

Complexity results by Tseng [14] imply, however, that deciding whether there exists a finite $\kappa$ for which a given matrix is $\mathcal{P}_{*}(\kappa)$ is an NP-hard problem. To be precise, Tseng [14] showed that the problem of deciding whether a given integer matrix is not sufficient is NP-complete in the Turing model.

In this paper we therefore also investigate semidefinite programming (SDP) based heuristics for computing a (finite) value $\kappa$ such that a given matrix $M$ is $\mathcal{P}_{*}(\kappa)$, if such a value exists. We show that our heuristic provides a suitable value of $\kappa$ for the so-called $\mathcal{P}$-matrices (where all principle minors are positive). 


\subsection{Outline of the paper}

Our paper is structured as follows. We first survey some matrix classes related to the $\mathcal{P}_{*}(\kappa)$ matrices in Sect. 2.1. Then we discuss the complexity of computing the handicap of a matrix if it is finite. We first show that the handicap can be exponential in the bit size of the matrix (Sect. 3), and discuss the complexity implications. Then we review an algorithm of Väliaho [16] for computing the handicap of a matrix exactly (if it is finite), and show that this algorithm requires $\Omega\left(6^{n}\right)$ operations (Sect. 4). These complexity results motivate the study of an SDP-based heuristic to compute an upper bound on the handicap in Sects. 5 and 6. Finally, we present some numerical examples for the heuristic in Sect. 7.

\subsection{Notation}

We use the following notation throughout the paper. Scalars, vectors, and indices are denoted by lowercase Latin letters, matrices by capital Latin letters, and sets by capital calligraphic letters. $\mathbb{R}_{+}^{n}$ denotes the nonnegative orthant of $\mathbb{R}^{n} ; I$ denotes the identity matrix of appropriate dimension, and $X$ is the diagonal matrix whose diagonal elements are the coordinates of the vector $x$, i.e. $X=\operatorname{diag}(\mathrm{x})$. The vector $x \circ s=X s$ is the componentwise product (Hadamard product) of the vectors $x$ and $s$. The $i$ th entry of a vector $x$ is denoted by $x_{i}$. If $A \in \mathbb{R}^{n \times n}, A_{\mathcal{J K}}$ denotes the submatrix of $A$ with rows indexed by the index set $\mathcal{J} \subset\{1, \ldots, n\}$ and columns by the index set $\mathcal{K} \subset\{1, \ldots, n\}$. We will always use $\mathcal{I}$ to denote the index set $\mathcal{I}:=\{1, \ldots, n\}$. We denote the vector of ones by $e$. If $f, g: \mathbb{N} \rightarrow \mathbb{R}_{+}$, we write that $f(n)=O(g(n))$ if $f / g$ is bounded from above by a constant, and $f(n)=\Omega(g(n))$ if $f / g$ is bounded from below by a constant.

\section{A review of matrix classes and complexity results}

\subsection{Matrix classes}

We start by recalling the well-known class of positive definite matrices.

Definition 2 A matrix $M \in \mathbb{R}^{n \times n}$ belongs to the class of positive definite matrices $(P D)$, if $x^{T} M x>0$ holds for all $x \in \mathbb{R}^{n} \backslash\{0\}$. Likewise, $M \in \mathbb{R}^{n \times n}$ belongs to the class of positive semidefinite matrices ( $P S D$ ) if $x^{T} M x \geq 0$ holds for all $x \in \mathbb{R}^{n}$. Furthermore, $M \in \mathbb{R}^{n \times n}$ is a skew-symmetric matrix ${ }^{1}(S S)$, if $x^{T} M x=0$ for all $x \in \mathbb{R}^{n}$.

By Definition $1, \mathcal{P}_{*}(0)$ is the class of positive semidefinite matrices (note that we do not require symmetry in the definition of positive semidefiniteness).

\footnotetext{
${ }^{1}$ Sometimes the skew-symmetric matrix is called antisymmetric according to its other definition: a matrix $M$ is skew-symmetric, if $M^{T}=-M$.
} 
Definition 3 A matrix $M \in \mathbb{R}^{n \times n}$ is called a $\mathcal{P}_{*}$-matrix if it is a $\mathcal{P}_{*}(\kappa)$-matrix for some $\kappa \geq 0$, i.e.

$$
\mathcal{P}_{*}=\bigcup_{\kappa \geq 0} \mathcal{P}_{*}(\kappa) .
$$

Definition 4 A matrix $M \in \mathbb{R}^{n \times n}$ is a column sufficient matrix if for all $x \in \mathbb{R}^{n}$

$$
X(M x) \leq 0 \text { implies } X(M x)=0,
$$

and row sufficient if $M^{T}$ is column sufficient. Matrix $M$ is sufficient if it is both row and column sufficient.

Kojima et al. [7] proved that any $\mathcal{P}_{*}$-matrix is column sufficient and Guu and Cottle [3] proved that it is row sufficient, too. Therefore, each $\mathcal{P}_{*}$-matrix is sufficient. Väliaho [15] proved the other direction of inclusion, thus the class of $\mathcal{P}_{*}$-matrices coincides with the class of sufficient matrices.

Definition 5 A matrix $M \in \mathbb{R}^{n \times n}$ is a $\mathcal{P}$-matrix, if all of its principal minors are positive.

Lemma 1 ([1]) The following properties for a matrix $M \in \mathbb{R}^{n \times n}$ are equivalent:

1. $M$ is a $\mathcal{P}$-matrix.

2. For every nonzero $x \in \mathbb{R}^{n}$ there is an index $i$ such that $x_{i}(M x)_{i}>0$.

Definition 6 A matrix $M \in \mathbb{R}^{n \times n}$ is a $\mathcal{P}_{0}$-matrix, if all of its principal minors are nonnegative.

In the sense of the next lemma, the closure of the matrix class $\mathcal{P}$ is $\mathcal{P}_{0}$.

Lemma 2 ([2]) The following properties for a matrix $M \in \mathbb{R}^{n \times n}$ are equivalent:

1. $M$ is a $\mathcal{P}_{0}$-matrix.

2. $M+\varepsilon I$ is a $\mathcal{P}$-matrix for every $\varepsilon>0$.

It is known [7] that the following relations hold among matrix classes $S S \subsetneq P S D \subsetneq$ $\mathcal{P}_{*} \subsetneq \mathcal{P}_{0}, \quad S S \cap \mathcal{P}=\emptyset, \quad P S D \cap \mathcal{P} \neq \emptyset, \quad \mathcal{P} \subsetneq \mathcal{P}_{*}, \quad \mathcal{P}_{*}\left(\kappa_{1}\right) \subsetneq \mathcal{P}_{*}\left(\kappa_{2}\right)$ for $\kappa_{1}<\kappa_{2}$, $\mathcal{P}_{*}(0) \equiv P S D$.

\subsection{Complexity of LCP for the various matrix classes}

We now consider the complexity of the LCP problem with integer data (1) in the Turing model of computation. To be precise, we have the decision problem (LCP): Given $n \in \mathbb{N}, M \in \mathbb{Z}^{n \times n}$ and $q \in \mathbb{Z}^{n}$, does (1) have a solution?

With reference to the matrix classes in Fig. 1, the known complexity results are summarized in Table 1.

It is important for our purposes to note that the complexity of LCP for $\mathcal{P}_{*}$-matrices is still open. In the next section we will return to this point. 


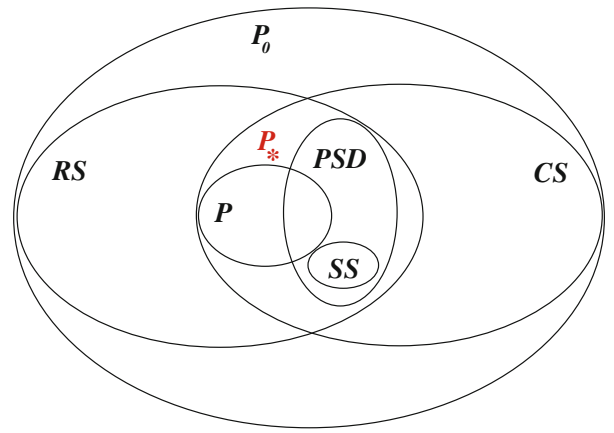

Fig. 1 The inclusion relations between various matrix classes; $C S$ column sufficient, $R S$ row sufficient, $S S$ skew-symmetric, $P S D$ positive semidefinite

Table 1 The complexity of the LCP problem with integer data in the Turing model, for various matrix classes

\begin{tabular}{lll}
\hline Matrix class & Complexity of LCP & Reference \\
\hline PSD & P & {$[6]$} \\
$\mathcal{P}$ & Not NP-hard, unless NP=co-NP & {$[10]$} \\
$\mathcal{P}_{*}$ & Unknown & \\
$\mathcal{P}_{0}$ & NP-complete & [7], p. 33 \\
\hline
\end{tabular}

\section{How large can the handicap $\hat{\kappa}(M)$ be in terms of $L(M)$ ?}

An important question is whether we can polynomially bound the handicap in terms of the bit size of the matrix. The answer is no, and the following matrix ${ }^{2}$ provides a counterexample.

$$
M=\left(\begin{array}{rrrrrrr}
1 & 0 & 0 & 0 & \ldots & 0 & 0 \\
-1 & 1 & 0 & 0 & \ldots & 0 & 0 \\
-1 & -1 & 1 & 0 & \ldots & 0 & 0 \\
& & & & & & \\
\vdots & & & & \ddots & & \\
-1 & -1 & -1 & -1 & \ldots & -1 & 1
\end{array}\right)
$$

Every principal submatrix of $M$ is a lower triangular matrix and it has an all-ones diagonal, so that its determinant is one. Therefore the matrix $M$ is a $\mathcal{P}$-matrix, and its handicap is finite. On the other hand, the matrix $M$ is not positive semidefinite, because, for example, for the all-ones vector $e$ the product $e^{T} M e$ is negative. Therefore the handicap has to be positive.

\footnotetext{
2 This matrix was suggested to us by Zsolt Csizmadia (private communication), as an example where the elements of principal pivotal transform of a given matrix cannot be polynomially bounded by the elements of the original matrix.
} 
Definition 7 A principal pivotal transformation of a matrix $A=\left(\begin{array}{ll}A_{\mathcal{J J}} & A_{\mathcal{J K}} \\ A_{\mathcal{K} \mathcal{J}} & A_{\mathcal{K} \mathcal{K}}\end{array}\right)$ where $\mathcal{J} \cup \mathcal{K}=\{1, \ldots, n\}$ and $A_{\mathcal{J} \mathcal{J}}$ is nonsingular, is the matrix

$$
\left(\begin{array}{cc}
A_{\mathcal{J} \mathcal{J}}^{-1} & -A_{\mathcal{J} \mathcal{J}}^{-1} A_{\mathcal{J K}} \\
A_{\mathcal{K} \mathcal{J}} A_{\mathcal{J} \mathcal{J}}^{-1} & A_{\mathcal{K} \mathcal{K}}-A_{\mathcal{K} \mathcal{K}} A_{\mathcal{J} \mathcal{J}}^{-1} A_{\mathcal{J K}}
\end{array}\right)
$$

We claim that the principal pivotal transformation of $M \in \mathbb{Z}^{n \times n}$ for the submatrix defined by the first $n-1$ rows and columns is:

$$
\tilde{M}=\left(\begin{array}{ccccccc}
1 & 0 & 0 & 0 & \ldots & 0 & 0 \\
1 & 1 & 0 & 0 & \ldots & 0 & 0 \\
2 & 1 & 1 & 0 & \ldots & 0 & 0 \\
4 & 2 & 1 & 1 & \ldots & 0 & 0 \\
\vdots & & & & \ddots & & \\
-2^{n-2} & -2^{n-3} & -2^{n-4} & -2^{n-5} & \ldots & -1 & 1
\end{array}\right) .
$$

Indeed, in this case $\mathcal{J}=\{1, \ldots, n-1\}$, and $M_{\mathcal{J} \mathcal{J}}$ has the same structure as $M$ does. Using the identity $\sum_{i=0}^{k-1} 2^{i}=2^{k}-1$, it is easy to check that

$$
M_{\mathcal{J} \mathcal{J}}^{-1}=\left(\begin{array}{ccccccc}
1 & 0 & 0 & 0 & \ldots & 0 & 0 \\
1 & 1 & 0 & 0 & \ldots & 0 & 0 \\
2 & 1 & 1 & 0 & \ldots & 0 & 0 \\
4 & 2 & 1 & 1 & \ldots & 0 & 0 \\
\vdots & & & & & & \\
2^{n-3} & 2^{n-4} & 2^{n-5} & 2^{n-6} & \ldots & 1 & 1
\end{array}\right)
$$

which proves our claim.

Theorem 1 ([3,7]) Let $M \in \mathbb{R}^{n \times n}$ be a sufficient matrix. Then:

1. The handicaps of $M$ and all its principal pivotal transforms are the same.

2. The handicap of $M$ is at least as large as that of any of its proper principal submatrices.

Accordingly $\hat{\kappa}(M) \geq \hat{\kappa}\left(\tilde{M}_{\mathcal{K} \mathcal{K}}\right)$, where $\mathcal{K}=\{1, n\}$. Using the closed form expression of Väliaho [16] for the handicap of a $2 \times 2$ matrix,

$$
\hat{\kappa}\left(\begin{array}{ll}
m_{11} & m_{12} \\
m_{21} & m_{22}
\end{array}\right)=\frac{1}{4}\left[\frac{m_{21}^{2}}{\left(\sqrt{m_{11} m_{22}}+\sqrt{m_{11} m_{22}-m_{12} m_{21}}\right)^{2}}-1\right]
$$


we obtain

$$
\hat{\kappa}\left(\tilde{M}_{\mathcal{K} \mathcal{K}}\right)=\hat{\kappa}\left(\begin{array}{cc}
1 & 0 \\
-2^{n-2} & 1
\end{array}\right)=\frac{1}{4}\left(-1+\frac{2^{2(n-2)}}{2^{2}}\right)=-0.25+2^{2 n-8} .
$$

It means, that $\hat{\kappa}(M)=\Omega\left(2^{2 n}\right)$, while all elements of matrix $M$ equal $-1,0$ or 1 . Since we can encode these elements of $M$ using at most $2 n^{2}$ bits, we have $\hat{\kappa}(M)=\Omega\left(2^{\sqrt{L}}\right)$, where $L$ is the bit size of $M$.

Remark 1 Consider the LCP problem (1) for $\mathcal{P}_{*}(\kappa)$ matrices. Our example shows that the typical $O((1+\kappa) \sqrt{n} L(M, q))$ iteration bound for interior point algorithms for these problems is not polynomial in $L(M, q)$ in general. Thus the Turing model complexity of the class of LCP's with $\mathcal{P}_{*}$-matrices remains open.

We close this section with a conjecture that the size of the handicap $\hat{\kappa}(M)$ is at most exponential in the bit size of the matrix $M$, and a related theorem.

Conjecture 1 There exists a univariate polynomial $p$ with the following property: for any (integer) sufficient matrix $M$ with encoding bit size $L(M)$, one has $\hat{\kappa}(M) \leq$ $2^{p(L(M))}$, i.e. there exists an upper bound on the handicap with encoding size polynomially bounded by the encoding size of the matrix.

In the conjecture, we do not make claims about the bit size of the handicap itself, since it can be an irrational number.

We now consider the computation complexity of the following decision problem:

Given integers $t, n>0$, and an integer $n \times n$ matrix $M$, is $\hat{\kappa}(M)>t$ ?

Theorem 2 The decision problem (5) is in NP in the Turing model.

Proof Assume $M \in \mathbb{Z}^{n \times n}$ with bit size $L(M)$. A 'yes' answer to the decision problem (5) has a certificate given by a suitable vector $x \in \mathbb{R}^{n}$ that satisfies

$$
(1+4 t) \sum_{i \in \mathcal{I}_{+}(x)} x_{i}(M x)_{i}+\sum_{i \in \mathcal{I}_{-}(x)} x_{i}(M x)_{i} \leq-1 .
$$

We now derive a new certificate from $x$ that has size polynomial in $L(M)$ and $t$, and can therefore be checked in polynomial time. For our given $x$, we define the index sets $\mathcal{I}_{+}(x)=\left\{1 \leq i \leq n: x_{i}(M x)_{i}>0\right\}$ and $\mathcal{I}_{-}(x)=\left\{1 \leq i \leq n: x_{i}(M x)_{i}<0\right\}$, as before, as well as

$$
\begin{aligned}
& \mathcal{I}_{++}(x):=\left\{i \in \mathcal{I}_{+}(x):(M x)_{i}>0 \text { and } x_{i} \geq 0\right\} \\
& \mathcal{I}_{--}(x):=\left\{i \in \mathcal{I}_{-}(x):(M x)_{i} \leq 0 \text { and } x_{i} \geq 0\right\}
\end{aligned}
$$

Let us now consider the following quadratic programming problem (in decision form): does there exist a $u \in \mathbb{R}^{n}$ such that:

$$
(1+4 t) \sum_{i \in \mathcal{I}_{+}(x)} u_{i}(M u)_{i}+\sum_{i \in \mathcal{I}_{-}(x)} u_{i}(M u)_{i} \leq-1,
$$


as well as

$$
\begin{aligned}
& u_{i} \geq 0, \quad(M u)_{i} \geq 0 \forall i \in \mathcal{I}_{++}(x), \\
& u_{i} \leq 0, \quad(M u)_{i} \leq 0 \forall i \in \mathcal{I}_{+}(x) \backslash \mathcal{I}_{++}(x), \\
& u_{i} \geq 0, \quad(M u)_{i} \leq 0 \forall i \in \mathcal{I}_{--}(x), \\
& u_{i} \leq 0, \quad(M u)_{i} \geq 0 \forall i \in \mathcal{I}_{-}(x) \backslash \mathcal{I}_{--}(x) ?
\end{aligned}
$$

By construction, a solution is given by $u=x$. Since the decision version of quadratic programming is in NP [17], there also exists a solution, say $\bar{u}$, with bit size bounded by a polynomial in $L(M)$ and $t$. Thus $\bar{u}$ is a suitable 'yes'-certificate for problem (5) that can be verified in polynomial time.

Theorem 3 If Conjecture 1 is true, then the decision problem (5) is NP-complete in the Turing model.

Proof Assume that $M \in \mathbb{Z}^{n \times n}$ of bit size $L(M)$ is given and that $M$ is not sufficient. If Conjecture 1 is true, we may obtain a 'yes' certificate or the decision problem (5) for $t:=\left\lceil 2^{p(L(M))}\right\rceil$. By Theorem 2 , the bit size of this certificate may be assumed to be polynomial in $L(M)$.

Thus we obtain a certificate that $M$ is not sufficient, and the certificate may be verified in polynomial time. Since deciding if a matrix is not sufficient is NP-complete in the Turing model (Corollary 1 in [14]), the proof is complete.

\section{The computational complexity of Väliaho's algorithm}

In this section we recall Väliaho's [16] recursive method to evaluate the handicap of a sufficient matrix, and we determine a lower bound on the complexity of this procedure. (Väliaho [16] gives no explicit analysis of the complexity of the algorithm in his paper.)

Let $M \in \mathbb{R}^{n \times n}$ be a $\mathcal{P}$-matrix, but not PSD. Väliaho [16] defined

$\hat{\kappa}_{n-1}:=\max \left\{\hat{\kappa}\left(B_{\mathcal{I}-i, \mathcal{I}-i}\right): B\right.$ is a principal pivotal transform of $M$ and $\left.i \in \mathcal{I}\right\}$, where $\mathcal{I}:=\{1, \ldots, n\}$ as before.

Let us assume that $\hat{\kappa}_{n-1}$ is known. If the matrix $M$ is sufficient but not a $\mathcal{P}$-matrix, then $\hat{\kappa}(M)=\hat{\kappa}_{n-1}[16$, Theorem 6.2]. Algorithm 1 computes the handicap of matrix $M$, if it is a $\mathcal{P}$-matrix.

In order to avoid repetition, Väliaho determines the handicap of $M$ by calculating the handicaps of all principal submatrices of order $i$ of all principal pivotal transforms of $M$ sequentially for $i=2, \ldots, n$. (Recall that there is a closed form for the handicap of two by two sufficient matrices (see (4)).

We denote the operational counts of

- determining the handicap of matrix $M$ by $k_{n}$, where $M \in \mathbb{R}^{n \times n}$, 


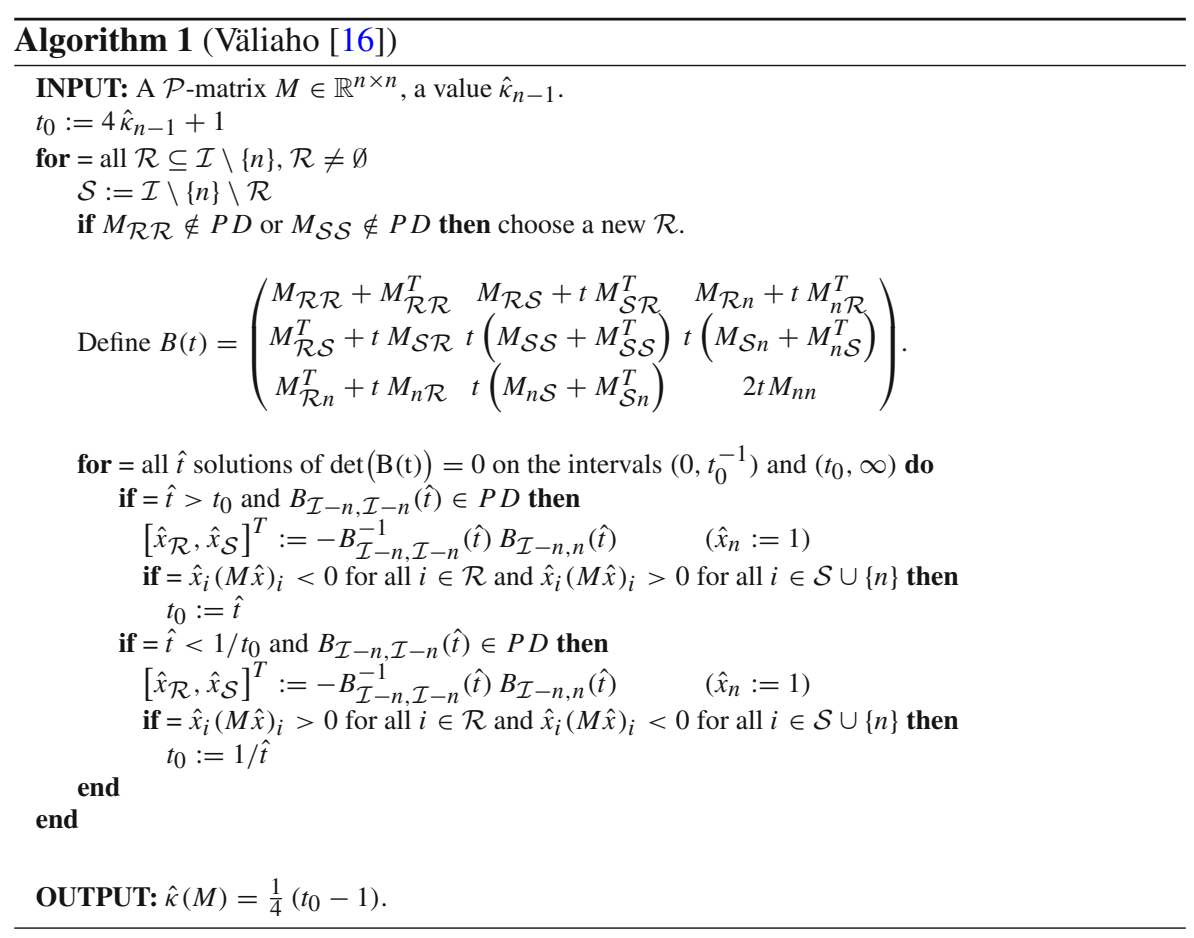

- solving for $t$ in $\operatorname{det}(\mathrm{B}(\mathrm{t}))=0$ by $d_{n}$, where $B(t) \in \mathbb{R}^{n \times n}$,

- determining $\hat{\kappa}_{n-1}(M)$ by $c_{n-1}$.

In Algorithm 1 we choose the set $\mathcal{R}$ as a subset of $\mathcal{I}-n$, but $\mathcal{R} \neq \varnothing$, so we have $2^{n-1}-1$ possibilities. Then

$$
k_{n} \geq\left(2^{n-1}-1\right) d_{n}+c_{n-1}
$$

To determine $c_{n-1}$ we have to calculate the handicap of $n-1$ dimensional matrices.

Now let us assume, that we know the handicap of all principal submatrices of order $i-1(i \geq 3)$ of all principal pivotal transforms of $M$. Then to determine the handicap of the principal submatrices of order $i$ of a principal pivotal transform of $M$ we need at least $\left(2^{i-1}-1\right) d_{i}$ operations. The matrix $M$ has $2^{n}-1$ proper pivotal transforms and each of them has $\left(\begin{array}{c}n \\ i\end{array}\right)$ principal submatrices of size $i$, so

$$
k_{n} \geq\left(2^{n-1}-1\right) d_{n}+\left(2^{n}-1\right) \sum_{i=2}^{n-1}\left(\begin{array}{l}
n \\
i
\end{array}\right)\left(2^{i-1}-1\right) d_{i}
$$

where $d_{i} \geq 2$, therefore

$$
k_{n} \geq 2^{n-1}+\left(2^{n}-1\right) \sum_{i=2}^{n-1}\left(\begin{array}{l}
n \\
i
\end{array}\right) 2^{i-1}
$$




$$
\begin{aligned}
& =2^{n-1}+\left(2^{n}-1\right) \frac{1}{2}\left[\sum_{i=0}^{n}\left(\begin{array}{l}
n \\
i
\end{array}\right) 2^{i}-\left(\begin{array}{l}
n \\
0
\end{array}\right) 2^{0}-\left(\begin{array}{l}
n \\
1
\end{array}\right) 2^{1}-\left(\begin{array}{l}
n \\
n
\end{array}\right) 2^{n}\right] \\
& =2^{n-1}+\left(2^{n}-1\right) \frac{1}{2}\left[3^{n}-1-2 n-2^{n}\right] \\
& \geq \frac{1}{5} 6^{n} \quad \text { if } n \geq 3 .
\end{aligned}
$$

The drawback of the algorithm of Väliaho is that no upper bound on the handicap is obtained before the algorithm terminates. Due to the complexity of $\Omega\left(6^{n}\right)$ the algorithm is therefore not practical if, say, $n \geq 15$. In fact, our computational experience with Algorithm 1 (see Sect. 7) is that it becomes impractical already for $n \geq 7$. This motivates our search for algorithms that can provide an upper bound on the handicap in a reasonable time in the remainder of the paper.

\section{Optimization models to compute the handicap}

Our aim is to determine the handicap of a sufficient matrix, or at least give an upper bound on it. For convenience, we define the handicap of a non-sufficient matrix as infinity.

We have already mentioned, that the class of positive semidefinite matrices coincides with $\mathcal{P}_{*}(0)$, so the handicap of a positive semidefinite matrix is 0 . The positive semidefiniteness can be determined in polynomial time. Indeed, the matrix $M \in P S D$ if and only if $M+M^{T} \in P S D$, and the latter one is already symmetric, so we need only check its eigenvalues. In the remainder of this section we construct different models whose optimal values are all equal to the handicap of the matrix $M$. For the simplicity, we write $\hat{\kappa}$ instead of $\hat{\kappa}(M)$. In this section we use subscripts for $\hat{\kappa}$ according from which model it is determined.

Considering Definition 1 we get the following problem whose optimal value ${ }^{3}$ is the handicap of the matrix $M$.

$$
\hat{\kappa}_{0}:=\inf \left\{\kappa \geq 0: q_{\kappa}(x) \geq 0, \forall x \in \mathbb{R}^{n}\right\}
$$

where

$$
q_{\kappa}(x):=x^{T} M x+4 \kappa \sum_{\mathcal{I}_{+}} x_{i}(M x)_{i} .
$$

The function $q_{\kappa}$ is not a polynomial, therefore we reformulate the problem. Let $\alpha_{j}(x)=\max \left\{0, x_{j}(M x)_{j}\right\}$, then $\sum_{\mathcal{I}_{+}} x_{i}(M x)_{i}=\sum_{j=1}^{n} \alpha_{j}(x)$. Using the new

\footnotetext{
3 The optimal value of problem (6) without the constraint $\kappa \geq 0$ can be negative for positive definite matrices, for example it will be -0.25 for the identity matrix. Since the handicap is a nonnegative value by definition, we add the constraint $\kappa \geq 0$.
} 
variables, our model is the following

$$
\hat{\kappa}_{1}:=\inf \left\{\kappa \geq 0: p_{\kappa}(x, \alpha) \geq 0, \forall(x, \alpha) \in \mathcal{K}_{1}\right\}
$$

where

$$
\begin{aligned}
p_{\kappa}(x, \alpha) & :=x^{T} M x+4 \kappa \sum_{i=1}^{n} \alpha_{i}, \quad \text { and } \\
\mathcal{K}_{1} & =\{(x, \alpha): \alpha \geq x \circ M x, \quad \alpha \geq 0\} .
\end{aligned}
$$

This is an equivalent model in the sense that $\hat{\kappa}_{0}=\hat{\kappa}_{1}$, moreover the feasible sets

$$
\begin{aligned}
& \mathcal{F}_{0}=\left\{\kappa \geq 0: q_{\kappa}(x) \geq 0 \forall x \in \mathbb{R}^{n}\right\} \quad \text { and } \\
& \mathcal{F}_{1}=\left\{\kappa \geq 0: p_{\kappa}(x, \alpha) \geq 0 \forall x \in \mathcal{K}_{1}\right\}
\end{aligned}
$$

coincide. The inclusion $\mathcal{F}_{1} \subseteq \mathcal{F}_{0}$ is a direct consequence of the idea behind the new variable $\alpha$. For the other direction, let $\kappa \in \mathcal{F}_{0}$ and $(x, \alpha) \in \mathcal{K}_{1}$, then

$$
p_{\kappa}(x, \alpha)=x^{T} M x+4 \kappa \sum_{i=1}^{n} \alpha_{i} \geq x^{T} M x+4 \kappa \sum_{\mathcal{I}_{+}} x_{i}(M x)_{i}=q_{\kappa}(x) \geq 0
$$

so $\kappa \in \mathcal{F}_{1}$.

The constant term of the polynomial $p_{\kappa}$ is zero, moreover

$$
p_{\kappa}(x, \alpha)=\|x\|^{2} p_{\kappa}\left(\frac{x}{\|x\|}, \frac{\alpha}{\|x\|^{2}}\right)
$$

for all $x \neq 0$. Therefore the following reformulation again has the same optimal value.

$$
\hat{\kappa}_{2}:=\inf \left\{\kappa \geq 0: p_{\kappa}(x, \alpha) \geq 0, \forall(x, \alpha) \in \mathcal{K}_{2}\right\},
$$

where

$$
\mathcal{K}_{2}=\{(x, \alpha):\|x\|=1, \quad \alpha \geq x \circ M x, \quad \alpha \geq 0\}
$$

The variable $\alpha$ can also be bounded.

$$
\hat{\kappa}_{3}:=\inf \left\{\kappa \geq 0: p_{\kappa}(x, \alpha) \geq 0, \forall(x, \alpha) \in \mathcal{K}_{3}\right\},
$$

where

$$
\mathcal{K}_{3}=\left\{(x, \alpha):\|x\|=1, \quad \alpha \geq x \circ M x, \quad\|\alpha\| \leq\|M\|_{2}, \quad \alpha \geq 0\right\} .
$$

Let us denote the feasible set of problem (8) and (9) by $\mathcal{F}_{2}$ and $\mathcal{F}_{3}$, respectively. Then $\mathcal{F}_{2} \subseteq \mathcal{F}_{3}$, because $\mathcal{K}_{3} \subseteq \mathcal{K}_{2}$. Furthermore, if $(x, \alpha) \in \mathcal{K}_{2}$, then $\|\alpha(x)\| \leq\|x \circ M x\| \leq$ 
$\|x\|\|M\|\|x\|=\|M\|_{2}$, thus $(x, \alpha(x)) \in \mathcal{K}_{3}$. Additionally, $p_{\kappa}(x, \alpha) \geq p_{\kappa}(x, \alpha(x))$, so $\mathcal{F}_{3} \subseteq \mathcal{F}_{2}$. We can summarize the above in the following lemma.

Lemma 3 Let $M \in \mathbb{R}^{n \times n}$, then the optimal value of the models (6), (7), (8) and (9) equal to the handicap of matrix $M$, namely $\hat{\kappa}_{0}=\hat{\kappa}_{1}=\hat{\kappa}_{2}=\hat{\kappa}_{3}=\hat{\kappa}(M)$.

Specifically, the problems (6), (7), (8) and (9) are infeasible if and only if the matrix $M$ is not sufficient.

Remark 2 The infima in problems (6), (7), (8) and (9) are always attained if $M$ is sufficient. Indeed, let $\left\{\kappa_{i}\right\} \in \mathcal{F}_{0}$ be such that $\lim _{i \rightarrow \infty} \kappa_{i}=\hat{\kappa}_{0}$. If $\hat{\kappa}_{0} \notin \mathcal{F}_{0}$, then there is a point $\bar{x}$ such, that $q_{\hat{\kappa}_{0}}(\bar{x})<0$. However, $q_{\kappa}(\bar{x})$ is a linear function of $\kappa$, thus $\lim _{i \rightarrow \infty} q_{\kappa_{i}}(\bar{x})=q_{\hat{\kappa}_{0}}(\bar{x})<0$, but this is a contradiction with $q_{\kappa_{i}}(\bar{x}) \geq 0$. The proof is similar for the other models.

\section{A hierarchy of SDP relaxations}

We will use the concept of Lasserre's paper [8], namely we give a hierarchy of SDP problems to compute the handicap. The optimal value of each SDP problem will be an upper bound on the handicap.

We recall some notation from the literature of real algebraic geometry. The ring of polynomials in the variables $z$, say, is denoted by $\mathbb{R}[z]$, and the cone of sum of squares of polynomials is denoted by $\Sigma$. The quadratic module generated by functions $g_{1}, \ldots, g_{m}$ is defined as

$$
M\left(g_{1}, \ldots, g_{m}\right)=\left\{s_{0}+\sum_{j=1}^{m} s_{j} g_{j}: s_{j} \in \Sigma, j=0, \ldots, m\right\} .
$$

It is easy to see, that if a polynomial is in the quadratic module $M\left(g_{1}, \ldots, g_{m}\right)$, then this polynomial is nonnegative on the set $\left\{z: g_{j}(z) \geq 0, j=1, \ldots, m\right\}$. For the other direction of implication, we need a special property of the quadratic module. The quadratic module $M\left(g_{1}, \ldots, g_{m}\right)$ is called Archimedean if there is a function $f \in M\left(g_{1}, \ldots, g_{m}\right)$ such that the set $\{z: f(z) \geq 0\}$ is compact.

Theorem 4 (Putinar [12]) Let $\mathcal{K}=\left\{z: g_{j}(z) \geq 0, j=1, \ldots, m\right\}$ and assume, that the quadratic module $M\left(g_{1}, \ldots, g_{m}\right)$ is Archimedean. For $p \in \mathbb{R}[z]$, if $p>0$ on $\mathcal{K}$ then $p \in M\left(g_{1}, \ldots, g_{m}\right)$.

Let us take into consideration the problem (9). In this case $\mathcal{K}=\mathcal{K}_{3} \subset \mathbb{R}^{2 n}$, and it is defined by $2 n+3$ functions: 


$$
\begin{aligned}
g_{i}(x, \alpha) & =\alpha_{i}-x_{i}(M x)_{i}, \quad i=1, \ldots, n, \\
g_{n+i}(x, \alpha) & =\alpha_{i}, \quad i=1, \ldots, n, \\
g_{2 n+1}(x, \alpha) & =1-\sum_{i=1}^{n} x_{i}^{2}, \\
g_{2 n+2}(x, \alpha) & =-1+\sum_{i=1}^{n} x_{i}^{2}, \\
g_{2 n+3}(x, \alpha) & =\|M\|_{2}^{2}-\sum_{i=1}^{n} \alpha_{i}^{2} .
\end{aligned}
$$

Let $f=g_{2 n+1}+g_{2 n+3} \in M\left(g_{1}, \ldots, g_{2 n+3}\right)$. For this function the set

$$
\left\{(x, \alpha) \in \mathbb{R}^{2 n}: 1+\|M\|_{2}^{2}-\|(x, \alpha)\|^{2} \geq 0\right\}
$$

is compact, so the quadratic module is Archimedean (see for example [9, p. 186]), thus

$$
p_{\kappa}(x, \alpha)>0 \text { on } \mathcal{K}_{3} \Longrightarrow p_{\kappa} \in M\left(g_{1}, \ldots, g_{2 n+3}\right)
$$

Note that we require the positivity of $p_{\kappa}$ as opposed to the nonnegativity requirement in (9). However, we prove in the next lemma that the model is still correct if we replace nonnegativity by positivity in the case of $\mathcal{P}$-matrices.

Lemma 4 Let $M$ be a $\mathcal{P}$-matrix. Then

$$
\hat{\kappa}(M)=\inf \left\{\kappa \geq 0: p_{\kappa}(x, \alpha)>0, \forall(x, \alpha) \in \mathcal{K}_{3}\right\}
$$

Proof Let

$$
\widehat{\mathcal{F}}_{3}=\left\{\kappa \geq 0: p_{\kappa}(x, \alpha)>0, \forall(x, \alpha) \in \mathcal{K}_{3}\right\}
$$

and remember $\mathcal{F}_{3}=\left\{\kappa \geq 0: p_{\kappa}(x, \alpha) \geq 0, \forall(x, \alpha) \in \mathcal{K}_{3}\right\}$. By definition, $\widehat{\mathcal{F}}_{3} \subset \mathcal{F}_{3}$, therefore $\inf _{\mathcal{F}_{3}} \kappa \leq \inf _{\widehat{\mathcal{F}}_{3}} \kappa$.

On the other hand, if $M$ is a $\mathcal{P}$-matrix, then $\sum_{\mathcal{I}_{+}} x_{i}(M x)_{i}>0$ for every nonzero $x$ by Lemma 1 . Thus $\sum_{i=1}^{n} \alpha_{i} \geq \sum_{\mathcal{I}_{+}} x_{i}(M x)_{i}>0$ for all $(x, \alpha) \in \mathcal{K}_{3}$. Therefore, if $p_{\kappa}(x, \alpha) \geq 0$ for some $(x, \alpha) \in \mathcal{K}_{3}$, then $p_{\kappa+\varepsilon}(x, \alpha)>0$ for all $\varepsilon>0$. In other words, ${ }^{4}$

$$
\kappa \in \mathcal{F}_{3} \quad \Longrightarrow \quad \kappa+\varepsilon \in \widehat{\mathcal{F}}_{3} \quad \forall \varepsilon>0
$$

According Remark 2,

$$
\hat{\kappa}=\min \left\{\kappa \geq 0: p_{\kappa}(x, \alpha) \geq 0, \forall(x, \alpha) \in \mathcal{K}_{3}\right\}
$$

\footnotetext{
4 The converse implication is also true in (11).
} 
namely $\hat{\kappa} \in \mathcal{F}_{3}$. Hence by (11), there is a sequence in $\widehat{\mathcal{F}}_{3}$, which tends to $\hat{\kappa}$ (for example, $\hat{\kappa}+1 / t, t \rightarrow \infty)$, so inf $\widehat{\mathcal{F}}_{3} \kappa \leq \hat{\kappa}$, which completes the proof.

Lemma 4 and the implication (10) mean, that if the matrix $M$ is a $\mathcal{P}$-matrix, then $\hat{\kappa}=\inf \left\{\kappa: p_{\kappa} \in M\left(g_{1}, \ldots, g_{2 n+3}\right)\right\}$. The quadratic module can be described with SDP constraints, however the dimension of the problem will be infinite. Therefore, a hierarchy of SDP relaxations is used that corresponds to a sequence of truncated SOS problems. In our case the truncated SOS problem in order $t$ is the following:

$$
\begin{aligned}
& \kappa^{(t)}:=\inf \kappa \\
& \text { s.t. } \\
& x^{T} M x+4 \kappa \sum_{i=1}^{n} \alpha_{i}=s_{0}(x, \alpha)+\sum_{j=1}^{n}\left(\alpha_{j}-x_{j}(M x)_{j}\right) s_{j}(x, \alpha) \\
& +\sum_{j=1}^{n} \alpha_{j} s_{n+j}(x, \alpha)+\left(\|M\|_{2}^{2}-\sum_{i=1}^{n} \alpha_{i}^{2}\right) s_{2 n+1}(x, \alpha) \\
& +\left(1-\sum_{i=1}^{n} x_{i}^{2}\right) r(x, \alpha) \\
& s_{j}(x, \alpha) \in \Sigma, \quad j=0, \ldots, 2 n+1 \\
& \operatorname{deg}\left(s_{0}\right) \leq 2 t \text {, } \\
& \operatorname{deg}\left(s_{j}\right) \leq 2 t-2, \quad j=1, \ldots, 2 n+1 \\
& r \in \mathbb{R}[x, \alpha], \operatorname{deg}(r) \leq 2 t-2 \\
& \kappa \geq 0 \text {. }
\end{aligned}
$$

We mention that the SDP formulation of problem (12) may be solved in $O\left(\left(\begin{array}{c}n+2 t \\ 2 t\end{array}\right)^{4.5} \ln (L / \epsilon)\right)$ operations to obtain an $\epsilon$-approximation of $\kappa^{(t)}$ by using interior point methods.

One can see, that the feasibility regions of the truncated SOS problems expand as $t$ increases, namely $\mathcal{F}^{(t)} \subseteq \mathcal{F}^{(t+1)}$, where $\mathcal{F}^{(t)}$ is the set of feasible solutions of truncated SOS problem in order $t$. Details on the SDP reformulation of SOS problems like problem (12) may be found in the paper by Lasserre [8], or in the more recent survey paper [9]. Here we only mention that the optimal value $\kappa^{(t)}$ may be computed to any fixed precision in polynomial time for fixed values of $t$ by using the interior point methods for SDP to solve an SDP reformulation of (12).

We can summarize the results of this section in the following theorem.

Theorem 5 Let $M \in \mathbb{R}^{n \times n}$ with handicap $\hat{\kappa}(M)(\hat{\kappa}(M):=\infty$ if $M$ is not sufficient), and let $\kappa^{(t)}$ be defined as in $(12)\left(\kappa^{(t)}=\infty\right.$ if problem (12) is infeasible). Then:

$-\kappa^{(t)}=\infty$ for all $t \in \mathbb{N}$ if $M$ is not sufficient;

$-\kappa^{(t)} \geq \kappa^{(t+1)} \geq \hat{\kappa}(M)$ if $\kappa^{(t)}$ is finite;

- $\hat{\kappa}(M)=\lim _{t \rightarrow \infty} \kappa^{(t)}$ if $M$ is a $\mathcal{P}$-matrix;

- $0=\hat{\kappa}(M)=\kappa^{(1)}$ if $M$ is PSD.

In words, if $\kappa^{(t)}$ is finite for some $t$, then this certifies that $M$ is sufficient. On the other hand, we only have the guarantee that $\kappa^{(t)}$ will be finite (for sufficiently large $t$ ) in 
the cases of PSD and $\mathcal{P}$-matrices. Moreover, in the case of $\mathcal{P}$-matrices, $\kappa^{(t)}$ may only become finite for large values of $t$, since it is NP-hard to decide if $M$ is a $\mathcal{P}$-matrix.

We will still show that $\kappa^{(1)}=\hat{\kappa}(M)$ if $M \in \mathbb{R}^{2 \times 2}$. Unfortunately, this nice property does not hold in higher dimensions: we will also present a $3 \times 3$ sufficient matrix for which the truncated SOS problem with order one is not feasible, i.e. where $\hat{\kappa}(M)$ is finite but $\kappa^{(1)}=\infty$.

It remains a topic for future research to try and obtain additional finite convergence results, i.e. $\kappa^{(t)}=\hat{\kappa}(M)$ for some finite value of $t$. However, finite convergence results are a bit problematic for the Lasserre hierarchy in general: results are only known for convex problems, and for problems where the feasible set is finite; see Laurent [9] for a survey of these results.

\subsection{The first order SOS relaxation}

For the first order Relaxation the polynomial $s_{0}$ in (12) is at most quadratic, and the $s_{i}(i=1, \ldots, 2 n+1)$ are nonnegative numbers and $r$ is a real number. By setting $(x, \alpha)=0$, we conclude that $s_{0}(0,0)+s_{2 n+1}+r=0$, so $r \leq 0$. Furthermore, there are no mixed terms $x_{i} \alpha_{j}$ on the left hand side of the first constraint in (12), and on the right hand side such a term can appear only in $s_{0}$. Therefore, $s_{0}(x, \alpha)=s_{01}(x)+s_{02}(\alpha)$, where $s_{01}$ and $s_{02}$ are also sums of squares and we can assume without loss of generality that $s_{02}(0)=0$. Similarly, on the left hand side there are no linear terms $x_{j}$, and on the right hand side such terms can only appear in $s_{01}$. Therefore $s_{01}(x)=s_{03}(x)+c^{2}$, where $s_{03}$ is also SOS, $s_{03}(0)=0$ and $c$ is a real number. Indeed, let $s_{01}=\sum q_{i}^{2}$, then every $q_{i}$ is linear. Let us decompose $q_{i}=\bar{q}_{i}+c_{i}$, where $\bar{q}_{i}(0)=0$ and $c_{i}$ is a real number. Thus $s_{01}=\sum\left(\bar{q}_{i}+c_{1}\right)^{2}=\sum \bar{q}_{i}^{2}+\sum c_{i}^{2}+2 \sum c_{i} \bar{q}_{i}$, where the last term has to be zero since $s_{01}$ is free of linear terms.

The first constraint of problem (12) has to hold for $\alpha=0$ as well, so we search for a quadratic SOS polynomial $s_{0}$, nonnegative numbers $s_{i}(i=1, \ldots, n, 2 n+1)$ and a real number $r$ such that the following equality holds for all $x$ :

$$
x^{T} M x=s_{03}(x)+c^{2}-\sum_{i=1}^{n} s_{i} x_{i}(M x)_{i}+s_{2 n+1}\|M\|_{2}^{2}+r\left(1-\sum_{i=1}^{n} x_{i}^{2}\right) .
$$

By rearranging the equality (13) and using that the sum of constant terms is zero, we get the following constraint:

$$
\sum_{i=1}^{n}\left(s_{i}+1\right) x_{i}(M x)_{i}=s_{03}(x)-r \sum_{i=1}^{n} x_{i}^{2} \forall x .
$$

Let $S$ be the diagonal matrix, whose $i$ th diagonal element is $s_{i}+1$. Then the left hand side of (14) is $x^{T} S M x$. Furthermore, it is a quadratic polynomial of $x$ and it has to be a SOS (recall $r \leq 0$ ), which equivalently means that the left hand side has to be nonnegative for all $x$. Summarizing these observations leads to the following lemma. 
Lemma 5 The first order $(t=1)$ SOS relaxation (12) is feasible if and only if there are positive numbers $d_{i}(i=1, \ldots, n)$ such that the matrix DM is PSD (we do not require the symmetry of the matrix), where $D=\operatorname{diag}\left(d_{1}, \ldots, d_{n}\right)$.

Proof We have already proved the 'only if' part.

Now let us assume that there is a positive diagonal matrix $D=\operatorname{diag}(d)$ such that $D M$ is PSD. If $\min d_{i}<1$, then let $\tilde{D}=1 /\left(\min d_{i}\right) D$, namely, $\tilde{D}=\operatorname{diag}(\tilde{d})$, where $\tilde{d}=1 /\left(\min d_{i}\right) d$. Otherwise, let $\tilde{D}=D$. Since $\min d_{i}>0$, the matrix $\tilde{D}$ is well defined, it is still positive diagonal matrix, and $\tilde{D} M$ is PSD. Then $x^{T} \tilde{D} M x$ is a nonnegative quadratic polynomial, so it is an SOS. Therefore

$$
\begin{aligned}
s_{0} & =x^{T} \tilde{D} M x, \\
s_{i} & =\tilde{d}_{i}-1 \quad i=1, \ldots, n, \\
\kappa & =0.25 \max _{i=1, \ldots, n} s_{i}, \\
s_{n+i} & =4 \kappa-s_{i} \quad i=1, \ldots, n, \\
s_{2 n+1} & =0, \\
r & =0
\end{aligned}
$$

is a feasible solution of the first order SOS relaxation.

Remark 3 It is easy to see directly, that if $D$ is a positive diagonal matrix such that $\min d_{i} \geq 1$ and $D M$ is $\operatorname{PSD}$, then $M$ is a $\mathcal{P}_{*}(\kappa)$-matrix with $\kappa=0.25\left(\max d_{j}-1\right)$. Indeed,

$$
\left(\max d_{j}\right) \sum_{\mathcal{I}_{+}} x_{i}(M x)_{i}+\sum_{\mathcal{I}_{-}} x_{i}(M x)_{i} \geq \sum_{i=1}^{n} d_{i} x_{i}(M x)_{i} \geq 0 \quad \forall x \in \mathbb{R}^{n}
$$

In connection with Lemma 5, one may ask whether the set of matrices, for which the first order relaxation is already feasible, is a convex cone. The answer is no, it is a cone, but not convex. Indeed, the set in question is

$$
\mathcal{H}=\left\{M \in \mathbb{R}^{n \times n}: \exists S \in \mathbb{R}^{n \times n} \text { positive diagonal matrix such that } S M \in P S D\right\} .
$$

This set contains the zero matrix and $\mathcal{H}$ is closed under positive scalar multiplication, so it is a cone. On the other hand, let

$$
\begin{gathered}
M_{1}=\left(\begin{array}{ccc}
1 & 0 & 10 \\
0 & 0 & 0 \\
0 & 0 & 1
\end{array}\right) \text { and } M_{2}=\left(\begin{array}{ccc}
9 & 0 & 0 \\
30 & 10 & 0 \\
0 & 30 & 9
\end{array}\right) . \\
\tilde{M}_{1}=\operatorname{diag}(1,1,25) \cdot M_{1}=\left(\begin{array}{ccc}
1 & 0 & 10 \\
0 & 0 & 0 \\
0 & 0 & 25
\end{array}\right), \quad \operatorname{eig}\left(\tilde{M}_{1}+\tilde{M}_{1}^{T}\right)=\{0,0,52\}, \text { so } M_{1} \in \mathcal{H} . \\
\tilde{M}_{2}=\operatorname{diag}(25,5,1) \cdot M_{2}=\left(\begin{array}{ccc}
225 & 0 & 0 \\
150 & 50 & 0 \\
0 & 30 & 9
\end{array}\right), \quad \operatorname{eig}\left(\tilde{M}_{2}+\tilde{M}_{2}^{T}\right)=\{0,62.3,505.7\},
\end{gathered}
$$


so $M_{2} \in \mathcal{H}$. The set $\mathcal{H}$ is convex if and only if $\mathcal{H}+\mathcal{H}=\mathcal{H}$ (since the set $\mathcal{H}$ is a cone). We show, that the sum of the previous two matrices does not belong to $\mathcal{H}$.

$$
M_{3}=M_{1}+M_{2}=\left(\begin{array}{ccc}
10 & 0 & 10 \\
30 & 10 & 0 \\
0 & 30 & 10
\end{array}\right)
$$

Let us assume, that there is a positive diagonal matrix $D=\operatorname{diag}\left(d_{1}, d_{2}, d_{3}\right)$ such that $D_{3}$ is $P S D$. As we have already mentioned, a matrix $A$ is PSD if and only if $A+A^{T}$ is PSD. Additionally, a symmetric matrix is PSD if and only if its all principal minors are nonnegative. Since

$$
D M_{3}+\left(D M_{3}\right)^{T}=\left(\begin{array}{lll}
20 d_{1} & 30 d_{2} & 10 d_{1} \\
30 d_{2} & 20 d_{2} & 30 d_{3} \\
10 d_{1} & 30 d_{3} & 20 d_{3}
\end{array}\right)
$$

the diagonal elements of $D M_{3}+\left(D M_{3}\right)^{T}$ are positive. From the two dimensional principal minors we have

$$
\begin{aligned}
& 4 d_{1} d_{2}-9 d_{2}^{2} \geq 0 \Longrightarrow 4 d_{1} \geq 9 d_{2}, \\
& 4 d_{2} d_{3}-9 d_{3}^{2} \geq 0 \Longrightarrow 4 d_{2} \geq 9 d_{3}, \\
& 4 d_{1} d_{3}-d_{1}^{2} \geq 0 \Longrightarrow 4 d_{3} \geq d_{1}
\end{aligned}
$$

From the last two inequalities we get that $d_{2} \geq 9 / 16 d_{1}$, but it is contrary to the first inequality, because $d_{i}(i=1,2,3)$ are positive numbers.

The matrix $M_{3}$ is a $\mathcal{P}$-matrix with handicap $\hat{\kappa}\left(M_{3}\right)=0.91886$ (we will show this numerically later on), so by Lemma 5, the first order relaxation is not feasible for every $\mathcal{P}$-matrix.

\subsubsection{Two dimensional problem}

Now let us examine the two dimensional case, i.e., $M \in \mathbb{R}^{2 \times 2}$. Without loss of generality we may assume that $\left|m_{12}\right| \leq\left|m_{21}\right|$. Recall from (4) that the handicap of a sufficient, but not PSD $2 \times 2$ matrix $M$ is

$$
\hat{\kappa}(M)=\frac{1}{4}\left[\frac{m_{21}^{2}}{\left(\sqrt{m_{11} m_{22}}+\sqrt{m_{11} m_{22}-m_{12} m_{21}}\right)^{2}}-1\right] .
$$

Let $s_{1}=4 \hat{\kappa}$ and $s_{2}=0$. We claim that the matrix $S M$ is PSD. It is trivial if the matrix $M$ is positive semidefinite (in this case $\hat{\kappa}=0$ ). Now let assume, that $M$ is sufficient but not PSD. We use the following equivalence in our proof:

$$
\left(\begin{array}{ll}
a & b \\
c & d
\end{array}\right) \in P S D \Longleftrightarrow \begin{gathered}
a, d \geq 0 \\
(b+c)^{2} \leq 4 a d .
\end{gathered}
$$


The diagonal elements of $S M$ are nonnegative, because $M$ is a sufficient matrix, so $m_{11}$ and $m_{22}$ are nonnegative numbers, and the diagonal elements of $\mathrm{S}$ are positive. The third inequality holds as well. Indeed:

$$
\left(s_{1}+1\right) m_{12}+m_{21}=\frac{m_{12} m_{21}^{2}+m_{21}\left(\sqrt{m_{11} m_{22}}+\sqrt{m_{11} m_{22}-m_{12} m_{21}}\right)^{2}}{\left(\sqrt{m_{11} m_{22}}+\sqrt{m_{11} m_{22}-m_{12} m_{21}}\right)^{2}},
$$

and, using the identity

$$
\begin{aligned}
& m_{12} m_{21} \\
& \quad=\left(\sqrt{m_{11} m_{22}}-\sqrt{m_{11} m_{22}-m_{12} m_{21}}\right)\left(\sqrt{m_{11} m_{22}}+\sqrt{m_{11} m_{22}-m_{12} m_{21}}\right),
\end{aligned}
$$

one obtains

$$
\begin{aligned}
& \left(\left(s_{1}+1\right) m_{12}+m_{21}\right)^{2} \\
& =\left(\frac{2 m_{21} \sqrt{m_{11} m_{22}}\left(\sqrt{m_{11} m_{22}}+\sqrt{m_{11} m_{22}-m_{12} m_{21}}\right)}{\left(\sqrt{m_{11} m_{22}}+\sqrt{m_{11} m_{22}-m_{12} m_{21}}\right)^{2}}\right)^{2} \\
& =4\left(s_{1}+1\right) m_{11} m_{22} .
\end{aligned}
$$

Lemma 6 Let $M \in \mathbb{R}^{2 \times 2}$ be a sufficient matrix. Then the first order SOS relaxation $((12)$ with $t=1)$ is feasible and its optimal value is $\kappa^{(1)}=\hat{\kappa}(M)$.

\section{Numerical examples}

In this section we present some preliminary computational results for computing the upper bound $\kappa^{(t)}$ from (12) on the handicap. We solved the problem (12) with Gloptipoly 3.4 [4] using the SDP solver SeDuMi 1.1R3 [13]. The return status of Gloptipoly can be $-1,0$ or 1 . The meaning of the status in our case is the following: status $=-1$ : The truncated SOS problem is infeasible or the SDP solver has numerical problems.

status $=0: \quad$ The value $\kappa^{(t)}$ is an upper bound on the handicap.

status $=1: \quad$ The value $\kappa^{(t)}$ equals the handicap.

It is important to note that status $=1$ means that a certificate is obtained that $\kappa^{(t)}$ equals the handicap. This certificate is obtained from the dual SDP problem of (12); the details are beyond the scope of this paper, but may be found in Sect. 6 of [9].

In Table 2 below, we present the status (s), the bound $\kappa^{(t)}$ from (12), and the computational time (in seconds) with different orders of relaxations $(t=1,2, \ldots)$ for seven different matrices. The matrices $M_{1}, M_{2}$ and $M_{3}$ are from Sect. 6.1, while $M_{4}$ and $M_{5}$ are related to the paper of Väliaho [16]:

$$
M_{4}=\left(\begin{array}{rrr}
4 & 1 & 1 \\
2 & 1 & -2 \\
-4 & 3 & 1
\end{array}\right) \quad \text { and } M_{5}=\left(\begin{array}{rrr}
4 & 1 & 2 \\
2 & 1 & -2 \\
-4 & 3 & 1
\end{array}\right)
$$


Table 2 Numerical results for computing handicap of the matrices $M_{1}, \ldots, M_{7}$

\begin{tabular}{|c|c|c|c|c|c|}
\hline & \multicolumn{4}{|l|}{ Order of SOS relaxation } & \multirow{2}{*}{$\begin{array}{l}\text { Väliaho's } \\
\text { algorithm }\end{array}$} \\
\hline & 1 & 2 & 3 & 4 & \\
\hline$M_{1}$ & $\begin{array}{l}\mathrm{s}=0 \\
\kappa^{(1)}=6 \\
0.2 \mathrm{~s}\end{array}$ & $\begin{array}{l}\mathrm{s}=0 \\
\kappa^{(2)}=6 \\
0.8 \mathrm{~s}\end{array}$ & $\begin{array}{l}\mathrm{s}=0 \\
\kappa^{(3)}=5.99 \\
19.3 \mathrm{~s}\end{array}$ & $\begin{array}{l}\mathrm{s}=0 \\
\kappa^{(4)}=5.99 \\
636.7 \mathrm{~s} \\
\end{array}$ & $\begin{array}{l}\hat{\kappa}=6 \\
0.1 \mathrm{~s}\end{array}$ \\
\hline$M_{2}$ & $\begin{array}{l}\mathrm{s}=0 \\
\kappa^{(1)}=6 \\
0.2 \mathrm{~s}\end{array}$ & $\begin{array}{l}\mathrm{s}=1 \\
\kappa^{(2)}=6 \\
0.6 \mathrm{~s}\end{array}$ & - & - & $\begin{array}{l}\hat{\kappa}=6 \\
0.3 \mathrm{~s}\end{array}$ \\
\hline$M_{3}$ & $\begin{array}{l}\mathrm{s}=-1 \\
\kappa^{(1)}=\infty \text { (infeasible) } \\
0.2 \mathrm{~s}\end{array}$ & $\begin{array}{l}\mathrm{s}=1 \\
\kappa^{(2)}=0.91886 \\
0.5 \mathrm{~s}\end{array}$ & - & - & $\begin{array}{l}\hat{\kappa}=0.91886 \\
0.3 \mathrm{~s}\end{array}$ \\
\hline$M_{4}$ & $\begin{array}{l}\mathrm{s}=0 \\
\kappa^{(1)}=0.08986 \\
0.1 \mathrm{~s}\end{array}$ & $\begin{array}{l}\mathrm{s}=1 \\
\kappa^{(2)}=0.08986 \\
0.4 \mathrm{~s}\end{array}$ & - & - & $\begin{array}{l}\hat{\kappa}=0.08986 \\
0.6 \mathrm{~s}\end{array}$ \\
\hline$M_{5}$ & $\begin{array}{l}\mathrm{s}=0 \\
\kappa^{(1)}=0.03987 \\
0.2 \mathrm{~s}\end{array}$ & $\begin{array}{l}\mathrm{s}=1 \\
\kappa^{(2)}=0.03987 \\
0.4 \mathrm{~s}\end{array}$ & - & - & $\begin{array}{l}\hat{\kappa}=0.03987 \\
0.6 \mathrm{~s}\end{array}$ \\
\hline$M_{6}$ & $\begin{array}{l}\mathrm{s}=0 \\
\kappa^{(1)}=15.75 \\
0.3 \mathrm{~s}\end{array}$ & $\begin{array}{l}\mathrm{s}=1 \\
\kappa^{(2)}=15.75 \\
138.7 \mathrm{~s}\end{array}$ & - & - & $\begin{array}{l}\hat{\kappa}=15.75 \\
1737.7 \mathrm{~s}\end{array}$ \\
\hline$M_{7}$ & $\begin{array}{l}\mathrm{s}=0 \\
\kappa^{(1)}=0.039866 \\
0.3\end{array}$ & $\begin{array}{l}\mathrm{s}=1 \\
\kappa^{(2)}=0.039866 \\
413.1 \mathrm{~s}\end{array}$ & - & - & $\begin{array}{l}- \\
>12 \mathrm{~h}\end{array}$ \\
\hline
\end{tabular}

The matrix $M_{6}$ is a 6-by-6 matrix of the type (3). The last matrix is the following

$$
M_{7}=\left(\begin{array}{ccccccc}
1 & 0 & 0 & 0 & -2 & 0 & 0 \\
0 & 1 & 0 & -1 & 0 & 0 & 0 \\
0 & 0 & 1 & 0 & 0 & 2 & -2 \\
0 & 1 & 0 & 1 & 0 & 0 & 0 \\
5 & 0 & 0 & 0 & 2 & 0 & 0 \\
0 & 0 & -1 & 0 & 0 & 2 & 4 \\
0 & 0 & -3 & 0 & 0 & -10 & 7
\end{array}\right)
$$

The final column in Table 2 gives the solution times for our own Matlab implentation of Algorithm 1, i.e. of the algorithm by Väliaho [16]. In the table, the value of the handicap is certified by the second order relaxation $\left(i . e . \kappa^{(2)}=\hat{\kappa}(M)\right.$ ) for all the examples except $M_{1}$. For the matrix $M_{1}$, one has $\kappa^{(1)}=\hat{\kappa}(M)=6$, but Gloptipoly fails to certify this. Moreover, the values $\kappa^{(3)}$ and $\kappa^{(4)}$ should equal 6 as well, and the inaccuracies seen in the table are due to limited accuracy in the solution of the underlying SDP problems. Also note that the running time increases exponentially with the order $t$ for $M_{1}$ - this is a consequence of the size of the underlying SDP problems, where the matrix variables are of order $n^{t}$. 
Note also, that Väliaho's algorithm (Algorithm 1) requires a prohibitive amount of time to compute the handicap, already if $n=7$.

In conclusion, computing the values $\kappa^{(t)}$ in (12) for small $t$ is a practical alternative to using the exact algorithm by Väliaho [16], if the goal is to obtain an upper bound on the handicap of a sufficient matrix.

Open Access This article is distributed under the terms of the Creative Commons Attribution Noncommercial License which permits any noncommercial use, distribution, and reproduction in any medium, provided the original author(s) and source are credited.

\section{References}

1. Fiedler, M., Pták, V.: On matrices with non-positive off-diagonal elements and positive principal minors. Czech. Math. J. 12, 382-400 (1962)

2. Guu, S.M.: Sign reversing and matrix classes. J. Optim. Theory Appl. 89(2), 373-387 (1996)

3. Guu, S.M., Cottle, R.W.: On a subclass of $P_{0}$. Linear Algebra Appl. 223(224), 325-335 (1995)

4. Henrion, D., Lasserre, J.-B., Löfberg, J.: Gloptipoly 3: moments, optimization and semidefinite programming. (2008) http://homepages.laas.fr/henrion/papers/gloptipoly3.pdf

5. Illés, T., Peng, J., Roos, C., Terlaky, T.: A strongly polynomial rounding procedure yielding a maximally complementary solution for $P_{*}(\kappa)$ linear complementarity problems. SIAM J. Optim. 11(2), 320-340 (2000)

6. Kojima, M., Mizuno, S., Yoshise, A.: A polynomial-time algorithm for a class of linear complementary problems. Math. Program. 44, 1-26 (1989)

7. Kojima, M., Megiddo, N., Noma, T., Yoshise, A.: A Unified Approach to Interior Point Algorithms for Linear Complementarity Problems. vol. 538 of Lecture Notes in Computer Science. Springer, Berlin (1991)

8. Lasserre, J.: Global optimization with polynomials and the problem of moments. SIAM J. Optimization. 11, 796-817 (2001)

9. Laurent, M.: Sums of squares, moment matrices and optimization over polynomials. In: Putinar, M., Sullivant, S., (eds.) Emerging Applications of Algebraic Geometry, vol. 149 in IMA Volumes in Mathematics and its Applications. Springer, pp. 157-270 (2009). Updated version available at: http:// homepages.cwi.nl/ monique/files/moment-ima-update-new.pdf

10. Megiddo, N.: A Note on the Complexity of P-Matrix LCP and Computing an Equilibrium. Research Report RJ 6439. IBM Almaden Research Center, San Jose, California (1988)

11. Potra, F.A., Liu, X.: Predictor-corrector methods for sufficient linear complementarity problems in a wide neighborhood of the central path. Optim. Methods Softw. 20(1), 145-168 (2005)

12. Putinar, M.: Positive polynomials on compact semi-algebraic sets. Indiana Univ. Math. J. 42, 969-984 (1993)

13. Sturm, J.F.: Using SeDuMi 1.02, a MATLAB toolbox for optimization over symmetric cones. Optim. Methods Softw. 11(12), 625-653 (1999)

14. Tseng, P.: Co-NP-completeness of some matrix classification problems. Math. Program. 88, 183-192 (2000)

15. Väliaho, H.: $P_{*}$-matrices are just sufficient. Linear Algebra Appl. 239, 103-108 (1996)

16. Väliaho, H.: Determining the handicap of a sufficient matrix. Linear Algebra Appl. 253, 279-298 (1997)

17. Vavasis, S.A.: Quadratic programming is in NP. Inf. Process. Lett. 36(2), 73-77 (1990) 